\title{
An Image Study on Local Anesthetic Spread in Healthy Volunteers Receiving Subcostal Exterior Semilunaris Transversus Abdominis Plane (SE-TAP) Block
}

\section{Qi Chen (iD) \\ Changyu Liang ${ }^{2}$ \\ Jingqiu Liang ${ }^{2}$ \\ Jiapeng Qiu (iD) ${ }^{3}$ \\ Bin Yang $\mathbb{D}^{3}$}

'Department of Anesthesiology, Chongqing University Cancer Hospital, Chongqing, People's Republic of China; ${ }^{2}$ Chongqing Cancer Multi-Omics Big Data Application Engineering Research Center, Chongqing University Cancer Hospital, Chongqing, People's Republic of China; ${ }^{3}$ Department of Anesthesiology, The First Affiliated Hospital of Xiamen University, Xiamen, People's Republic of China
Correspondence: Bin Yang Department of Anesthesiology, The First Affiliated Hospital of Xiamen University, Xiamen, People's Republic of China Email yangbin4332@outlook.com
Background: Despite the popularity of the ultrasound-guided transversus abdominis plane (TAP) block and the diversity of advancing approaches, the extent of injectate spread limits its clinical benefits. This study used three-dimensional computed tomography (3D-CT) imaging and a cold stimulus to evaluate the spread of a local anesthetic injected through the subcostal exterior semilunaris to transversus abdominis plane (SE-TAP) block in healthy volunteers.

Methods: Eight healthy volunteers received a right-side ultrasound-guided SE-TAP block with $25 \mathrm{~mL}$ of $0.3 \%$ ropivacaine. The sensory block was assessed by a cold stimulus at 30 min, $2 \mathrm{~h}, 4 \mathrm{~h}$, and $8 \mathrm{~h}$ following the SE-TAP block according to the 18-zone division method. A CT scan and 3D imaging were performed after the first sensory assessment.

Results: The injectate spread into the transversus abdominis space in all eight volunteers. 3D imaging confirmed that the injectate spread extensively along the costal margin in the plane of the transverse abdominis muscle and that it surpassed the semilunaris. Regarding the assessment using cold stimulus, five of six anterior zones and three of six lateral zones obtained successful rates of cutaneous sensory block higher than 75\% 30 min after SE-TAP. Sensory block was achieved in the ventral dermatomes of all volunteers.

Conclusion: Our study showed that the SE-TAP injectate, which was administered using simple anatomical landmarks to provide reliable analgesia for abdominal surgery, consistently spread along the costal margin and extensively blocked cutaneous sensitivity in the anterior and lateral abdominal walls.

Keywords: subcostal exterior semilunaris, transversus abdominis plane block, threedimensional computed tomography

\section{Introduction}

In the last decade, ultrasound-guided transversus abdominis plane (TAP) block has been widely used as a technique for abdominal analgesia after surgery. ${ }^{1}$ As the technique is simple and the target site is relatively easy to identify, this application has rapidly expanded to address anesthetic and analgesic needs in gastroenterology, obstetrics, and gynecology surgeries. ${ }^{1-3}$ Despite the popularity of the technique, the extent of local anesthetic diffusion attributable to each approach remains unclear. ${ }^{4-6}$ This uncertainty limits its use in upper and extensive abdominal surgeries. 
The subcostal exterior semilunaris approach (SE-TAP) was first described in 2020 and involves advancing the needle with the in-plane approach from lateral to medial aiming toward the lateral border of the semilunar. ${ }^{7}$ This approach potentially blocks the anterior cutaneous branches of T6-T8 at the surface of the transverse abdominal muscle below the ribs, and it potentially spreads laterally to the lateral and anterior cutaneous branches of T9-T12. This single injection point has the potential of achieving broader analgesia of the lateral and anterior abdominal walls than any other approach or needle injection site.

It is important to unravel the characteristics of this block in terms of its anesthetizing capacity in the dermatomes T6-L2. To better characterize the spread of the SETAP block, we performed a unilateral SE-TAP block in healthy volunteers to assess the cutaneous sensory block area and to observe the spread of the injectate through three-dimensional computed tomography (3D-CT) imaging. Based on previous research, our main hypothesis was that, with SE-TAP, the local anesthetic would bypass the semilunaris to the cephalic side to reach the posterior rectus sheath of the rectus abdominis, and that it would also spread caudally to the mid-axillary line.

\section{Methods}

\section{Participants}

This study was approved by the ethics committee of the Chongqing University Cancer Hospital and registered in the
Clinical Trial Registry (http://www.chictr.org.cn) with registration number: ChiCTR2000038008. The registration date was Sep 9, 2020 and volunteer enrollment date was Nov 3, 2020. Written consent was obtained before all the procedures and it was conducted in accordance with the Declaration of Helsinki. Eight volunteers were recruited to participate. Inclusion criteria were age over 18 years old and full skin sensitivity in the abdomen as assessed by a cold stimulation test. Exclusion criteria included allergy to ropivacaine, claustrophobia, metal implants, BMI $<18 \mathrm{~kg} / \mathrm{m}^{2}$ or $>30 \mathrm{~kg} / \mathrm{m}^{2}$, history of abdominal surgery, and insulin-dependent diabetes.

\section{Ultrasound-Guided SE-TAP}

On the day of the study, the participants received the SETAP block at the surgical area of the hospital. After intravenous access were secured and the standard monitors were connected, the volunteers were placed in the supine position with no sedatives. An experienced anesthesiologist (A.S) placed a linear (6-12 MHz) ultrasound probe (Mindray M7 super, Shenzhen, China) inferior and parallel to the left costal margin and scanned along the oblique subcostal line to visualize the semilunaris along the costal margin. Four muscles (rectus, external oblique, internal oblique, and transversus abdominis) were identified (Figure 1A). Following skin infiltration with $2 \mathrm{~mL}$ of $2 \%$ lidocaine, we inserted an 5$\mathrm{cm} 22-\mathrm{G}$ needle (Braun, Aschaffenburg, Germany) with the in-plane approach from lateral to medial, aiming toward the lateral border of the semilunar along the costal margin

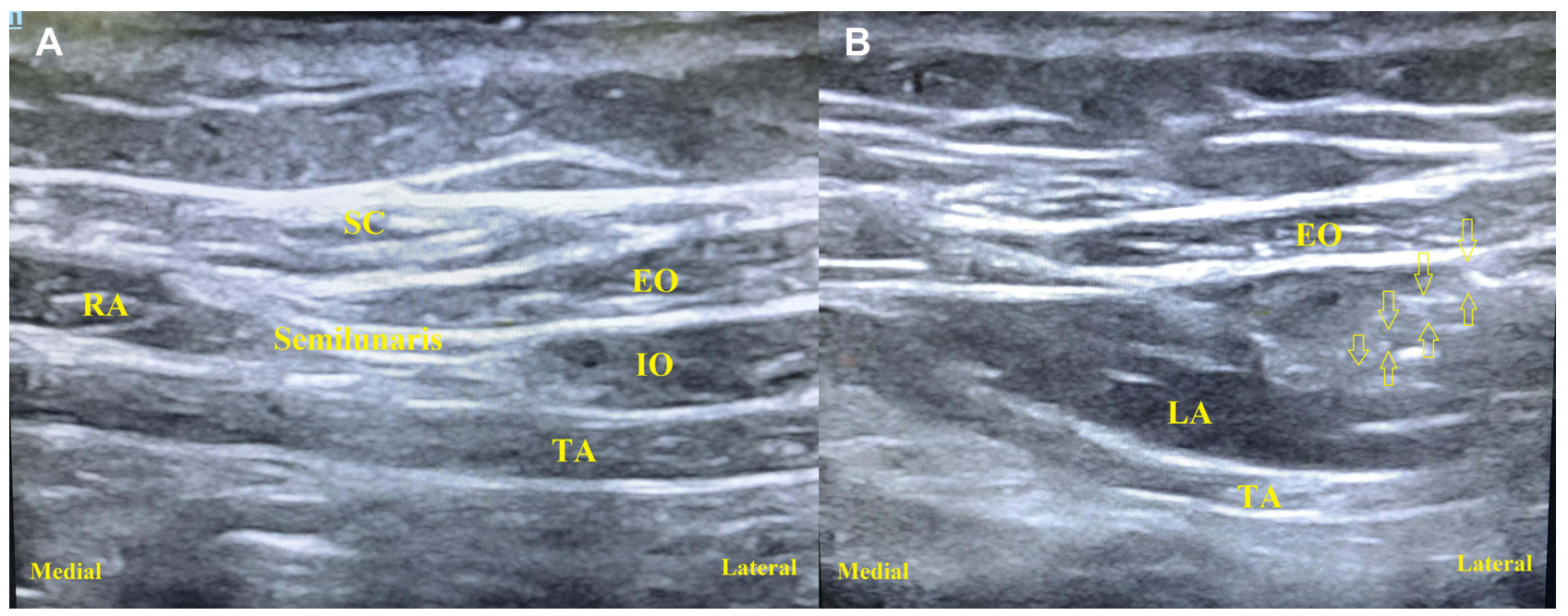

Figure I Sonograms of the muscle fascial structures near semilunaris and SE-TAP block. Arrow head points to needle used for anesthetic administration. (A) Four muscles (rectus, external oblique, internal oblique, and transversus abdominis) were identified. (B) After injection of $0.5 \mathrm{~mL}$ of saline into the fascial plane to confirm the presence of the needle tip in the target plane, $25 \mathrm{~mL}$ of $0.3 \%$ ropivacaine were injected in one side

Abbreviations: SC, subcutaneous tissue; EO, external oblique muscle; IO, internal oblique muscle; TA, transversus abdominal muscle; RA, rectus abdominal muscle; LA, local anesthetics. 


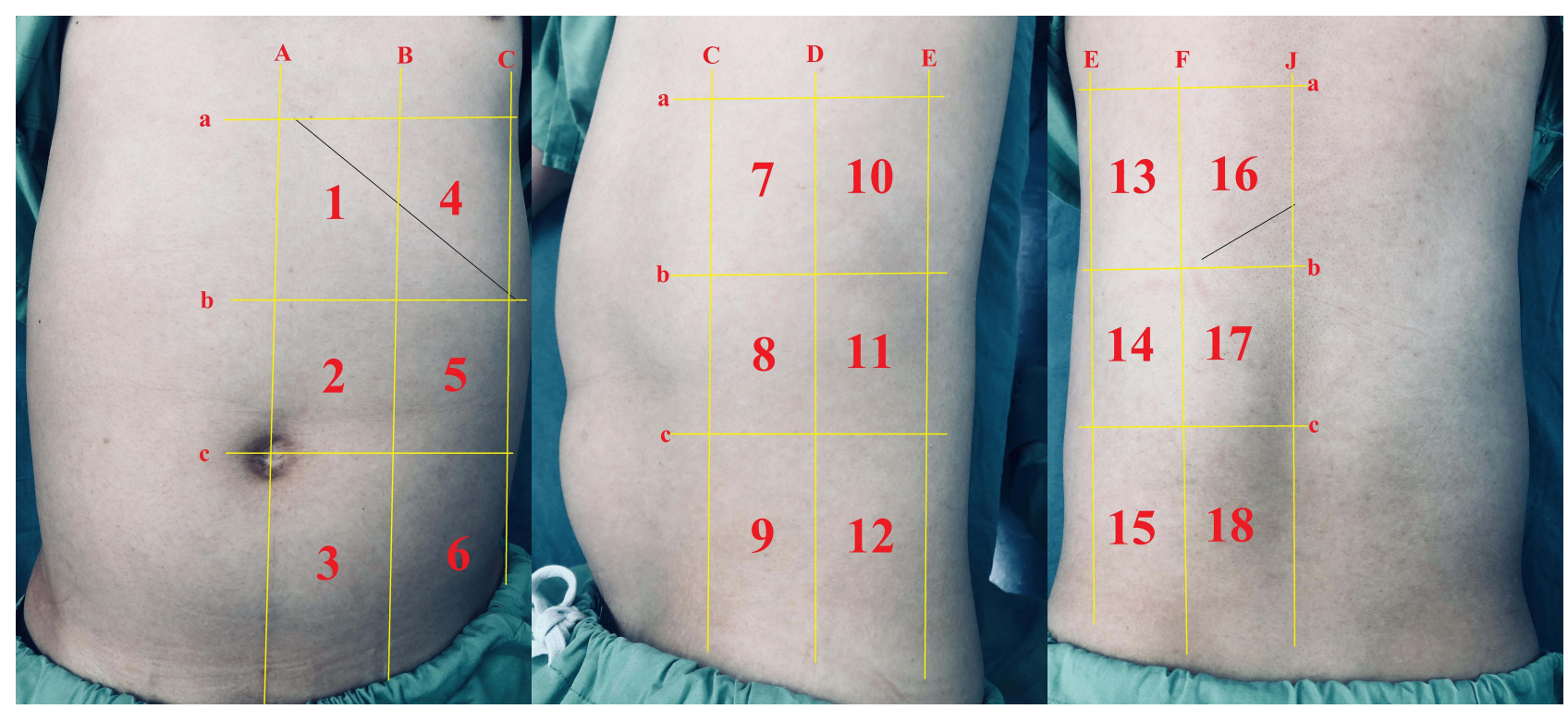

Figure 2 Detailed division of eighteen dermal zones. Anterior, lateral and posterior abdominal wall cover six dermal zones, respectively. A: vertical lines at the midline; B: mid-clavicular line; C: anterior axillary line; D: mid-axillary line; E: posterior axillary line; F: infrascapular line; J: back midline; a: by four horizontal lines at the xiphoid level; b: 12th costal level; c: umbilical level.

between the internal oblique and transversus abdominis muscles. After injection of $0.5 \mathrm{~mL}$ of saline into the fascial plane to confirm the presence of the needle tip in the target plane, $25 \mathrm{~mL}$ of $0.3 \%$ ropivacaine (AstraZeneca, Cambridge, United Kingdom) were injected in one side (Figure 1B).

\section{Assessment of Sensory Block and Analgesic Effect}

Volunteers were assessed $30 \mathrm{~min}, 2 \mathrm{~h}, 4 \mathrm{~h}$, and $8 \mathrm{~h}$ after the injection. The primary outcome was the cutaneous sensory block area (CSBA) and the secondary outcome was the 3D area. The abdominal CSBA was assessed (by one investigator) using a cold stimulus (ice cube). We used a dichotomous method to assess the cutaneous sensory block (successful block/failed block; identified by the application of the cold stimulus). We defined a block as successful when the cold sensation was lost or markedly reduced compared with the correspondent area of the nonblocked contralateral side. We defined a block as failed when the cold sensation was reduced but there was still sensitivity or when there was a persistence of the normal sensation. The 18 specific dermal zones used in this study (Figure 2) were described by vertical lines at the midline (A), mid-clavicular line (B), anterior axillary line (C), mid-axillary line (D), posterior axillary line (E), infrascapular line $(\mathrm{F})$, and back midline $(\mathrm{J})$, and by four horizontal lines at the xiphoid level (a), 12th costal level (b), umbilical level (c). ${ }^{8}$
We recorded the CSBA according to these 18 dermal zones 30 min, $2 \mathrm{~h}, 4 \mathrm{~h}$, and $8 \mathrm{~h}$ after the end of the block.

\section{CT Scan and Three-Dimensional Reconstruction}

After the first cold stimulus evaluation, a CT scan was performed using a long spine coil that covered the spine levels T8-L5 with a slice thickness of $3 \mathrm{~mm}$. All images were reviewed and analyzed by the same neuroradiologist experienced in CT image evaluation. The threedimensional reconstructions were made by VITA works (Shihui Med, Shanghai, China) to show the extent of drug diffusion and the contrast structures (eg, transversus abdominis, intercostal muscle, and ribs; Figure 3).

\section{Statistical Analysis}

Statistical analyses were performed using SPSS (version 22.0; SPSS Inc., Chicago, IL, USA). Qualitative variables (sensory block and effective rate) were evaluated using $\chi 2$ test with fisher correction. Quantitative variables (age, body mass index) were evaluated using the Student's $t$-test. A p-value $<0.05$ was considered statistically significant and demographics were presumed to follow a Gaussian distribution and are expressed as mean (SD).

\section{Results}

Six male volunteers and two female volunteers aged between 22 and 37 years old (average 30.3, SD 6.2) 

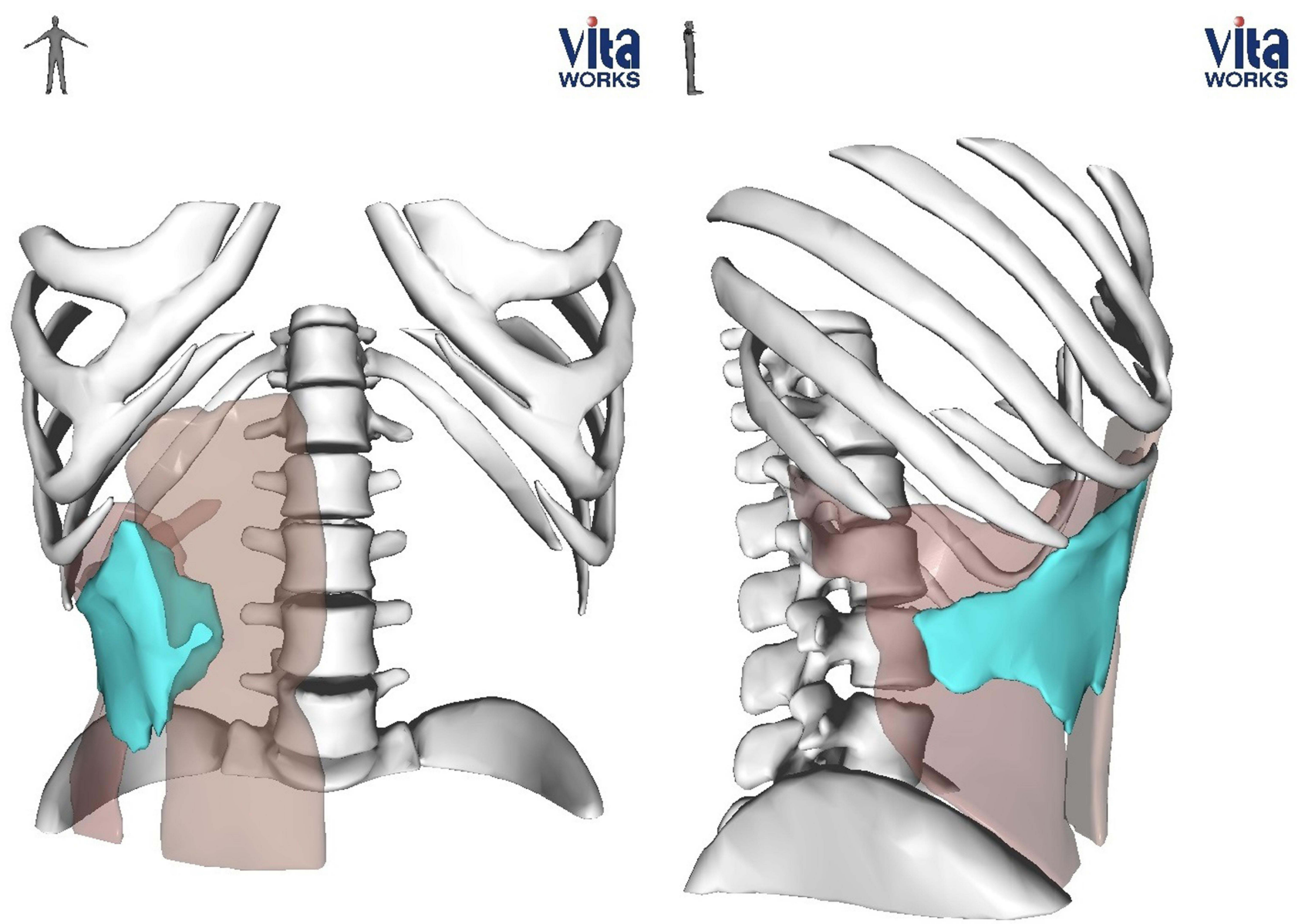

Figure 3 Three-dimensional reconstruction of CT scan for one volunteer after the first cold stimulus evaluation. Light brown area: transverse and rectus abdominis; bright blue area: the spread of local anesthetics.

were recruited for this study with demographic data as follows: weight, $59.4(\mathrm{SD}, 9.3) \mathrm{kg}$; height, $1.68(\mathrm{SD}$, $0.19) \mathrm{m}$; and body mass index 23.5 (SD, 4.2$) \mathrm{kg} / \mathrm{m} 2$.

In this study, all volunteers received a SE-TAP block under ultrasound guidance according to the study protocol. All procedures resulted in unilateral cutaneous block in the anterior and lateral abdominal walls. There were no cutaneous effects on the control side in any of the cases. The injectate spread extensively to the anterior and lateral abdominal walls compared with the unblocked side. The rates of successful cutaneous sensory block of the different zones at $30 \mathrm{~min}, 2 \mathrm{~h}, 4 \mathrm{~h}$, and $8 \mathrm{~h}$ are shown in Figure 4. The rates of successful cutaneous sensory block of zones 2 and 5 were always higher than $75 \%$ at all times, and in zones 3 and 8 they were higher than $75 \%$ within 4 h. Moreover, zones 1, 2, 3, 4, 5, 8, 9, and 11 obtained rates of successful cutaneous sensory block higher than 87.5\% 30 min after SE-TAP. The three-dimensional reconstructions showed that the anesthetic spread down and back along the costal margin, reaching the mid-axillary line and spreading across the semilunaris to the midline at the same time.

\section{Discussion}

Our study demonstrated that the ultrasound-guided SETAP block, with a low volume of local anesthetic, produces a widespread consistent cutaneous sensory block in the anterior and lateral abdominal walls based on the dermal zones method, but with some variation on the extent of the anesthesia. In addition, we visually observed the diffusion of the drug through a 3D reconstruction of CT results and confirmed that the drug spreads along the costal margin and through the semilunaris.

Some clinical trials have demonstrated that several approaches to TAP block (lateral, subcostal, posterior) can successfully anesthetize the anterior or lateral abdominal walls by showing a reduction in postoperative pain and in opioid consumption after abdominal surgery. ${ }^{1,2,9}$ 


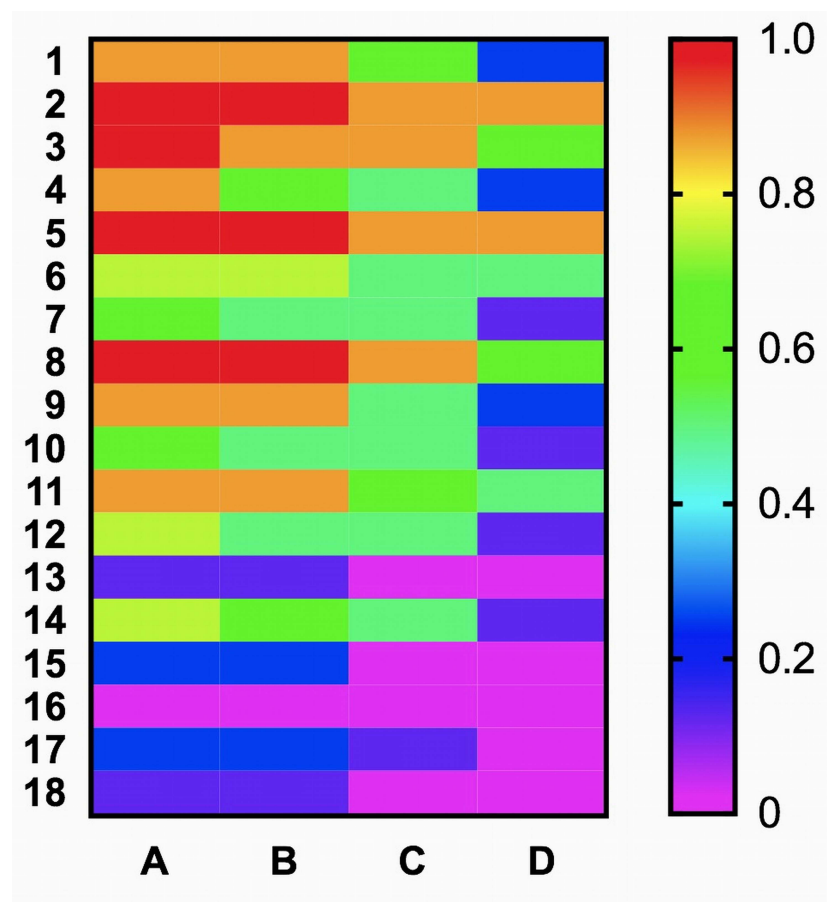

A: $30 \min \quad B: 2 h \quad C: 4 h \quad D: 8 h$

Figure 4 Rates of successful cutaneous sensory block of 18 dermal zones on the right abdomen at four time points after SE-TAP. A: 30min; B: $2 \mathrm{~h}$; C: $4 \mathrm{~h}$; D: $8 \mathrm{~h}$.

Notwithstanding, there is some controversy about the effectivity of TAP block. Ghisi and Oh et al found that ultrasoundguided lateral TAP blocks do not reduce opioid consumption after total laparoscopic hysterectomy or laparoscopic colorectal cancer surgery. ${ }^{10,11}$ Furthermore, a volunteer study by Juhl et al demonstrated that single-injection lateral TAP blocks produced only small areas of effective cutaneous sensory anesthesia with large variations in the anterior abdominal wall and moderate-to-poor reproducibility. ${ }^{12}$ Rozen et al claimed that if the TAP injection is not positioned under this distinct fascia, the block might only affect the nerves penetrating the part fascia layer. ${ }^{13}$

In the present SE-TAP study, we observed an effect on the anterior cutaneous branches of the lower thoracic nerves and on the branches innervating the anterior abdominal wall. These findings suggest that SE-TAP has a more precise and uniform position of the needle (aiming to the vertical crossing point at the angle formed by the lateral border of the semilunaris and the costal margin) and therefore exerts a significant cutaneous sensory block of the abdominis. 3D imaging also proved that the drug spreads well along the costal margin in the plane of the transverse abdominis muscle. Zones 2, 3, 5, and 8 achieved $100 \%$ effective cutaneous anesthesia 30 min after SE-TAP, and zones 1, 4, 9, and 11 achieved 87.5\% successful rates of cutaneous sensory block at the same point in time.

In upper abdominal surgery or abdominal surgery with large incisions, the bilateral dual TAP block is often used. $^{14,15}$ It is because of the resistance of the semilunar to the diffusion of drugs that lateral and subcostal approaches to TAP block are used in combination. The lateral approach is mainly to block the area of the middle and lower abdomen and the lateral abdominal wall while subcostal approaches is to block the upper abdomen and anterior abdominal wall. However, this method also brings the disadvantages of increased number of punctures and higher volumes of injected drugs. ${ }^{16}$ In addition, in order to increase the spread of the anesthetics, some researchers use a $10-\mathrm{cm}$ or even a $20-\mathrm{cm}$ needle to puncture the semilunar during the oblique subcostal TAP block and to inject the drug on the plane of the transverse abdominis. $^{17}$ In our study, $100 \%(8 / 8)$ of the volunteers' abdominal 3D imaging showed that the drug crossed the semilunar line from the lateral and reached between the rectus abdominis and the transversus abdominis muscles. Therefore, it is possible to inject the drug next to the semilunar instead of using a long needle to puncture it, which would cause more trauma and would require more skilled technique.

Clinically, whether it is open surgery, large incision surgery, or laparoscopic surgery with multiple small incisions and drainage tubes in different positions in the abdomen, our study reveals how the subcostal exterior semilunaris approach diffuses laterally and towards the middle, which can be a guide to provide anesthesia and pain management more precisely.

Our study has several limitations. Further clinical trials are needed to confirm the reliability of this approach because this sample size of volunteers in this study is small. Although cold and pain perception are associated with different sensory pathways in terms of receptors, conduction, and central integration, we used the cold stimulus because of it is relative comfort for volunteers. Furthermore, Børglum et al confirmed that pain and cold sensitivity share cutaneous areas, and that the absence of either of these sensations can be used to confirm the presence of sensory block. ${ }^{18}$ Because of the adverse effects of contrast media, radiopaque contrast agents have not been added to local anesthetics in the volunteer study. However, in this study, accurate CT-3D imaging can also show the drug diffusion range three-dimensionally and precisely.

\section{Conclusions}

Our study reveals that the subcostal exterior semilunaris approach to TAP block, which was performed using 
simple and fixed anatomical landmarks, could provide reliable, widespread cutaneous blocking from lateral to midline. Further studies are encouraged to validate this technique for analgesia after abdominal surgery.

\section{Data Sharing Statement}

Since this was a volunteer study, we have disclosed all data in this study with their consent.

\section{Acknowledgments}

We would like to thank Jing Ye, M.D, PhD, from the Nanfang Hospital Nanfang Medical University; Jihong Jiang, M.D, PhD, for Shanghai General Hospital of Shanghai Jiaotong University for their invaluable contribution to this study.

\section{Funding}

This work was supported by the High-Level Medical Personnel Training Project of Chongqing (2019GDRC017 to Bin Yang) and Scientific Technological Research Program of Chongqing Municipal Education Commission (KJQN201800104 to Qi Chen).

\section{Disclosure}

The authors declare no conflicts of interest.

\section{References}

1. Tran DQ, Bravo D, Leurcharusmee P, et al. Transversus Abdominis Plane Block: a Narrative Review. Anesthesiology. 2019;131 (5):1166-1190. doi:10.1097/ALN.000000000002842

2. Hong KY, Kim DK, Park HJ, et al. Analgesic Efficacy of Preemptive Transversus Abdominis Plane Block in Patients Undergoing Laparoscopic Colorectal Cancer Surgery. J Clin Med. 2020;9 (5): 1577 . doi: $10.3390 / \mathrm{jcm} 9051577$

3. Chen Q, Liu X, Zhong XJ, et al. Addition of dexmedetomidine or fentanyl to ropivacaine for transversus abdominis plane block: evaluation of effect on postoperative pain and quality of recovery in gynecological surgery. J Pain Res. 2018;16(11):2897-2903. doi:10.2147/ JPR.S178516

4. Carney J, Finnerty O, Rauf J, et al. Studies on the spread of local anaesthetic solution in transversus abdominis plane blocks. Anaesthesia. 2011;66:1023-1030. doi:10.1111/j.1365-2044.2011.06855.x

5. Stoving K, Rothe C, Rosenstock CV, et al. Cutaneous sensory block area, muscle-relaxing effect, and block duration of the transversus abdominis plane block: a randomized, blinded, and placebo-controlled study in healthy volunteers. Reg Anesth Pain Med. 2015;40:355-362. doi:10.1097/AAP.0000000000000252
6. Furuya T, Kato J, Yamamoto Y, et al. Comparison of dermatomal sensory block following ultrasound-guided transversus abdominis plane block by the lateral and posterior approaches: a randomized controlled trial. J Anaesthesiol Clin Pharmacol. 2018;34:205-210. doi:10.4103/joacp.JOACP_295_15

7. Chen Q, Jiang JH, Yang B. Modified thoracoabdominal sensory nerves block through subcostal exterior semilunaris approach (SE-TAP) provide broader analgesia for abdominal surgery. $J$ Clin Anesth. 2020;68:110077.

8. Ma J, Jiang Y, Tang S, et al. Analgesic efficacy of ultrasound-guided subcostal transversus abdominis plane block. Medicine (Baltimore). 2017;96(10):e6309. doi:10.1097/MD.0000000000006309

9. Faiz SHR, Alebouyeh MR, Derakhshan P, et al. Comparison of ultrasound-guided posterior transversus abdominis plane block and lateral transversus abdominis plane block for postoperative pain management in patients undergoing cesarean section: a randomized double-blind clinical trial study. $J$ Pain Res. 2018;11:5-9. doi:10.2147/JPR.S146970

10. Ghisi D, Fanelli A, Vianello F, et al. Transversus abdominis plane block for postoperative analgesia in patients undergoing total laparoscopic hysterectomy: a randomized, controlled, observer-blinded trial. Anesth Analg. 2016;123:488-492. doi:10.1213/ANE.0000000000001267

11. Oh TK, Yim J, Kim J, et al. Effects of preoperative ultrasound-guided transversus abdominis plane block on pain after laparoscopic surgery for colorectal cancer: a double-blind randomized controlled trial. Surg Endosc. 2017;31:127-134. doi:10.1007/s00464-016-4941-7

12. Juhl CS, Rothe C, Støving K, et al. Intraindividual variation of the transversus abdominis plane block: an exploratory study in healthy volunteers. Reg Anesth Pain Med. 2020;45(6):419-423. doi:10.1136/ rapm-2020-101322

13. Rozen W, Tran T, Ashton M, et al. Refining the course of the thoracolumbar nerves: a new understanding of the innervation of the anterior abdominal wall. Clin Anat. 2008;21:325-333. doi:10.1002/ca.20621

14. Tanggaard K, Jensen K, Lenz K, et al. A randomised controlled trial of bilateral dual transversus abdominis plane blockade for laparoscopic appendicectomy. Anaesthesia. 2015;70(12):1395-1400. doi:10.1111/anae.13234

15. Zhang J, Liu T, Zhou H, et al. The Safety and Efficacy of Ultrasound-Guided Bilateral Dual Transversus Abdominis Plane (BD-TAP) Block in ERAS Program of Laparoscopic Hepatectomy: a Prospective, Randomized, Controlled, Blinded, Clinical Study. Drug Des Devel Ther. 2020;14:2889-2898. doi:10.2147/DDDT.S255385

16. Børglum J, Maschmann C, Belhage B, et al. Ultrasound-guided bilateral dual transversus abdominis plane block: a new four-point approach. Acta Anaesthesiol Scand. 2011;55:658-663. doi:10.1111/ j.1399-6576.2011.02430.x

17. Hebbard PD, Barrington MJ, Vasey C. Ultrasound-guided continuous oblique subcostal transversus abdominis plane blockade: description of anatomy and clinical technique. Reg Anesth Pain Med. 2010;35:436-441. doi:10.1097/AAP.0b013e3181e66702

18. Børglum J, Jensen K, Christensen AF, et al. Distribution patterns, dermatomal anesthesia, and ropivacaine serum concentrations after bilateral dual transversus abdominis plane block. Reg Anesth Pain Med. 2012;37:294-301. doi:10.1097/AAP.0b013e31824c20a9 


\section{Publish your work in this journal}

The Journal of Pain Research is an international, peer reviewed, open access, online journal that welcomes laboratory and clinical findings in the fields of pain research and the prevention and management of pain. Original research, reviews, symposium reports, hypothesis formation and commentaries are all considered for publication. The manuscript management system is completely online and includes a very quick and fair peer-review system, which is all easy to use. Visit http:// www.dovepress.com/testimonials.php to read real quotes from published authors.

Submit your manuscript here: https://www.dovepress.com/journal-of-pain-research-journal 